\title{
LPAR1 regulates the development of intratumoral heterogeneity in ovarian serous cystadenocarcinoma by activating the PI3K/AKT signaling pathway
}

\author{
Ran Cui, Guangming Cao, Huimin Bai and Zhenyu Zhang*
}

\begin{abstract}
Background: To explore the role of lysophosphatidic acid receptor 1 (LPAR1) and its correlation with the PI3K/AKT pathway in the development of intratumoral heterogeneity (ITH) in human ovarian serous cystadenocarcinoma (OSC).

Methods: Immunohistochemical staining was performed to detect LPAR1 expression in matched primary and recurrent lesions from the same patients. Cell models of ITH were established using the limiting dilution methodology and Transwell invasion/migration assays. LPAR1 expression in the ITH cell models was silenced or upregulated with lentiviral particles, and the biological characteristics were evaluated using various in vitro and in vivo assessments of cell function. The levels of phosphorylated PI3K/AKT (p-PI3K/p-AKT) in LPAR1 knockdown and LPAR1-overexpressing cells were detected.

Results: The H-scores for LPAR1 staining in the lymphatic metastatic and recurrent lesions were noticeably higher than in the primary tumor lesions from the same patients $(P=0.024 / 0.031)$. High LPAR1 expression was associated with worse progression-free survival and overall survival $(P=0.017 / 0.039)$. Biological functions in vitro, including invasion, migration, and proliferation, and tumor formation in vivo were decreased in the LPAR1-silenced cells (all $\mathrm{P}<0.05)$. These cellular functions were significantly increased in the LPAR1-overexpressing cells in vitro and in vivo (all P<0.05). The levels of p-PI3K and p-AKT were significantly decreased in the LPAR1 knockdown cells and significantly increased in the LPAR1-overexpressing cells (all $P<0.05$ ).
\end{abstract}

Conclusions: Higher levels of the LPAR1 protein were associated with a poor prognosis. LPAR1 plays essential roles in the invasion, migration, and proliferation of heterogeneous subsets of OSC cell lines and the development of ITH of OSC, possibly by modulating the activity of the PI3K/AKT signaling pathway.

Keywords: Ovarian serous cystadenocarcinoma, Intratumoral heterogeneity, LPAR1, PI3K/AKT pathway, Single-cell subclones

\footnotetext{
*Correspondence: bhmdoctor@sina.com; zhenyuzhang2000@163.com Department of Obstetrics and Gynecology, Beijing Chao-Yang Hospital, Capital Medical University, No.8, North Road of Workers Stadium, Chaoyang District, Beijing 100020, China
} 


\section{Background}

Ovarian cancer is the most lethal gynecological carcinoma, and limited improvements in the survival of patients with advanced-stage ovarian cancer have been achieved over the last few decades [1]. Tumor metastasis, drug resistance and recurrence are the major causes of a poor prognosis in patients [2,3]. Within a single tumor, cancer cells display various heterogeneous features, including different biological characteristics, gene expression levels, and differentiation statuses; this phenomenon is referred to as intratumoral heterogeneity (ITH) [4-6]. ITH appears to be related to tumor metastasis, therapeutic resistance and recurrence, which lead to treatment failure in many human malignant tumors [7-11].

In our previous study, two pairs of single-cell subclones (named as A-H/A-L and S-H/S-L) with distinct invasive/migratory capacities were isolated and established from the same human ovarian serous cystadenocarcinoma (OSC) cell lines (SKOV3 and A2780) using the limiting dilution methodology [11, 12]; therefore, these cell lines have the same genetic backgrounds [11]. Compared with the A-L/S-L cells, the A-H/S-H cells exhibited significantly more aggressive phenotypes with respect to their biological functions in vitro and tumor formation in vivo. These two pairs of singlecell subclones are considered ideal cell models of ITH in human OSC. RNA-Seq and bioinformatics analyses revealed that the expression of components of the PI3K/AKT pathway, including lysophosphatidic acid receptor 1 (LPAR1), was significantly increased in the A-H/S-H cells. LPAR1 was the first identified highaffinity receptor for lysophosphatidic acid (LPA) and belongs to the $G$ protein-coupled receptor (GPCR) superfamily [13]. LPAR1 binds to and activates three types of $G$ proteins $\left(G_{\alpha i / o} o, 13 G_{\alpha q / 11}\right.$, and $\left.G_{\alpha 12 / 13}\right)$, which convey signals through downstream molecules and signaling pathways, including the PI3K/AKT pathway [13-15]. LPAR1 might play a vital role in various benign and malignant diseases by activating the PI3K/ AKT pathway [16-18]. Based on the results of our previous study and a literature review, we hypothesized that LPAR1 might be involved in the development of ITH in human OSC by activating the PI3K/AKT pathway. This study aimed to explore the role of LPAR1 and its correlation with the PI3K/AKT pathway in the development of ITH.

\section{Materials and methods}

The principal materials and instruments used in the present study are listed in Additional file 1: Table S1.

\section{Clinical data and tissue specimens}

After obtaining approval from the Beijing Chao-Yang Hospital Ethics Committee, patients with OSC who were diagnosed with high-grade serous carcinoma (HGSC) based on pathological examination and underwent cytoreductive surgery and standard platinum/ paclitaxel chemotherapy as the initial treatment at Beijing Chao-Yang Hospital between April 2006 and May 2015 were included in the present study. Additionally, matched paraffin-embedded tissues from these patients were collected from the pathology centre. The tissue specimens, including primary tumor lesions, abdominal disseminated lesions at presentation, lymphatic metastatic lesions at presentation and recurrent lesions, were all obtained from the same patients if possible. The four types of OSC tissue specimens from the same patients were matched for the detection of the heterogeneous expression of LPAR1 and an exploration of ITH in OSC. The clinical and pathological data for these patients were retrospectively collected and reviewed. The stages were reassessed based on the 2014 International Federation of Gynecology and Obstetrics (FIGO) system [19]. The progression-free survival (PFS) was calculated from the date of surgery to the date of recurrence; women who were disease-free at the time of their last visit were censored. The overall survival (OS) time was calculated from the date of surgery to the date of patient death from the disease, and patients who died from other conditions and survivors at the time of their last visit were censored.

\section{Tissue microarray (TMA)}

TMAs of matched primary tumor lesions, abdominal disseminated lesions, lymphatic metastatic lesions and recurrent lesions obtained from the same patients with OSC were constructed. The pathology slides were reexamined by two independent gynecological pathologists to confirm the diagnosis of OSC, and the accurate locations of the tumors were marked on the paraffinembedded tissue samples. Round tissue samples with a diameter of 1 millimetre were obtained from the tumor located in the donor block using a manual tissue array instrument (TMArrayer), and transferred into the TMA block $(10 \times 12$ arrays). Sequential sections were cut from the paraffin-embedded TMA blocks at a thickness $4 \mu \mathrm{m}$ and placed on blank slides.

\section{Immunohistochemical (IHC) staining}

IHC staining was performed as previously described [20]. After baking the tissue sections at $70{ }^{\circ} \mathrm{C}$ for $60 \mathrm{~min}$, the sections were deparaffinized, rehydrated, treated with $3 \%$ hydrogen peroxide to block endogenous peroxidase 
activity, and subjected to antigen retrieval (citrate buffer solution). After blocking with $10 \%$ goat serum at room temperature for $60 \mathrm{~min}$, tissue sections were incubated with the primary antibody (anti-LPAR1; $1: 100$ ) at $4{ }^{\circ} \mathrm{C}$ overnight. After an incubation with a horseradish peroxidase-conjugated goat anti-rabbit antibody for $60 \mathrm{~min}$, tissue sections were subjected to diaminobenzidine staining for color development. Subsequently, sections were subjected to hematoxylin counterstaining and dehydration, and then sealed with neutral resin.

\section{Evaluation of the IHC staining of the TMA}

A digital pathological section scanner (Pannoramic MIDI/P250) was utilized to capture images of the TMA slides, and the images were displayed at 1 to $400 \times$ magnification using Pannoramic Viewer 1.15.4 software. All images were independently evaluated by two independent gynecological pathologists who were blinded to the clinical data. A histochemistry score (H-score) based on a combination of the percentage of stained cells and staining intensity was calculated for the semiquantitative analysis. $\mathrm{H}$-score $=\sum$ (percentage $[0-100 \%] \times$ intensity $[1-3])=($ percentage of cells with weak intensity $\times 1)+($ percentage of cells with moderate intensity $\times 2)+($ percentage of cells with strong intensity $\times 3$ ) [21]. The differences in LPAR1 expression among the four types of OSC lesions from the same patients were compared. In addition, the relationship between LPAR1 expression and the patients' prognosis was analyzed. A poor prognosis was defined as PFS for less than 12 months or OS for less than 36 months.

\section{Cell culture and establishment of cell models of ITH}

Based on our previous study, two pairs of single-cell subclones with distinct invasive/migratory capacities (named A-H/A-L/S-H/S-L) derived from SKOV3 and A2780 cells may develop heterogeneous changes due to repeated passaging [11]. Thus, single-cell subclones were reisolated from $\mathrm{A}-\mathrm{H} / \mathrm{S}-\mathrm{H}$ and $\mathrm{A}-\mathrm{L} / \mathrm{S}-\mathrm{L}$ cells using the limiting dilution methodology as described in detail in our previous study. The A-H/S-H and A-L/S-L cells were cultured in RPMI-1640 supplemented with 10\% fetal bovine serum (FBS) and antibiotics $(100 \mathrm{U} / \mathrm{ml}$ penicillin and $0.1 \mathrm{mg} /$ $\mathrm{ml}$ streptomycin) at $37^{\circ} \mathrm{C}$ in a humidified incubator with a $5 \% \mathrm{CO}_{2}$ atmosphere. During the logarithmic growth phase, A-H, A-L, S-H, and S-L cells were diluted to $10 \mathrm{cells} / \mathrm{ml}$ and inoculated in 96-well plates with $100 \mu \mathrm{l}$ per well. At 4 to $6 \mathrm{~h}$ after inoculation, single adherent cells were observed under a microscope, and these cells were marked for continuous daily observation. Once the cells had proliferated to a certain density, single-cell subclones were successively transferred to 48-, 24-, and 6 -well plates and then culture dishes; subsequently, the cells were conventionally cultured. Transwell invasion/ migration assays were performed to select single-cell subclones with distinct invasive/migratory capacities, which were used as cell models of ITH in vitro. For further comparative analyses, single-cell subclones from similar generations within the 20th generation were used to avoid heterogeneous changes due to repeated passaging [11].

\section{Transwell invasion/migration assays}

The Transwell invasion/migration assays were performed using previously described method [22]. The Transwell chambers were either coated (invasion assay) or uncoated (migration assay) with $40 \mu \mathrm{l}$ of Matrigel diluted 1:10 in serum-free RPMI-1640. Cells suspended in $200 \mu \mathrm{l}$ of RPMI-1640 supplemented with $0.1 \%$ FBS were seeded in the upper portions of the chambers at a density of $8 \times 10^{4}$ cells/well (invasion assay) or $4 \times 10^{4}$ cells/well (migration assay). RPMI-1640 (600 $\mu \mathrm{l})$ supplemented with $10 \%$ FBS, which was regarded as a chemotactic factor, was added to the lower portions of the chambers. After $24-36 \mathrm{~h}$ of incubation at $37^{\circ} \mathrm{C}$, cells were fixed with paraformaldehyde (4\%) and stained with crystal violet. The noninvading/nonmigrating cells on the upper surface of the filter were carefully and completely removed with cotton swabs, and then the Transwell insert was observed and photographed under a microscope. ImagePro Plus software was used to calculate the number of stained cells on the lower surface of the membrane. Each assay was performed in triplicate.

\section{Cell proliferation assay with Counting Kit-8 (CCK-8)}

Cells $\left(3 \times 10^{3} /\right.$ well, 6 wells $)$ were seeded in 96-well plates in quadruplicate. The CCK- 8 reagent was added to four 96-well plates after incubations for 4, 24, 48, or $72 \mathrm{~h}$. After $2 \mathrm{~h}$ of incubation with the CCK- 8 reagent, the absorbance was measured at $450 \mathrm{~nm}$ using an enzymelinked immunosorbent assay plate reader. The assay was performed in triplicate.

\section{Western blotting}

Western blotting was performed as previously described [11]. The total protein was extracted from cells in the logarithmic growth phase using radioimmunoprecipitation assay lysis buffer containing protease and phosphatase inhibitors. Proteins (30 $\mu \mathrm{g}$ per group) were separated using $10 \%$ sodium dodecyl sulfate-polyacrylamide gel electrophoresis and transferred to a nitrocellulose membrane. After blocking with 5\% nonfat milk at room temperature for $2 \mathrm{~h}$, the nitrocellulose membrane was incubated with the primary antibody overnight at $4{ }^{\circ} \mathrm{C}$. The primary antibodies are listed in Additional file 2: Table S2. After an incubation with a horseradish 
peroxidase-conjugated secondary IgG antibody (1:3000) at room temperature for $2 \mathrm{~h}$, the nitrocellulose membrane was visualized using electrochemiluminescence (Bio-Rad GelDoc EZ), and the images were semi-quantitatively analyzed using ImageJ software. Each assay was performed in triplicate.

\section{Quantitative real-time polymerase chain reaction ( $q R T-P C R$ )} qRT-PCR was performed as previously described [11]. Total RNA was extracted from cells in the logarithmic growth phase using TRIzol reagent. The RNA concentration was determined using a NanoDrop ND-2000 spectrophotometer. Then, cDNAs were synthesized using a PrimeScript RT Reagent Kit with gDNA Eraser. qRT-PCR was performed using an ABI Prism 7500 RT-PCR system. The relative mRNA levels were normalized to levels of the endogenous control (GAPDH) and calculated using the $2^{-\Delta \mathrm{CT}}$ comparison method. The following primers were used: LPAR1 forward, 5'-CTTTGCTGGGTTGGC CTACTT-3', and reverse, 5'-GCCATGTGCTAACAG TCAGTCT-3', and GAPDH forward, 5'-AAGGTCATC CCTGAGCTGAAC-3', and reverse, 5'-ACGCCTGCT TCACCACCTTCT-3'. Each assay was performed in triplicate.

\section{Production and transduction of lentiviral particles}

Three specific short hairpin RNAs (shRNAs) targeting the LPAR1 gene, as well as a scrambled shRNA (negative control, NC), were designed and synthesized (Additional file 3: Table S3). Separate fragments containing different shRNAs targeting LPAR1 and the scrambled shRNA sequence were cloned into the GV248 plasmid. Subsequently, the GV248 plasmid and other packaging plasmids were cotransfected into HEK293T cells using a Lipofectamine 2000, and the viral particles were collected $48 \mathrm{~h}$ after transfection. After collecting cells infected with viral particles and extracting proteins and RNAs, the viral particles containing the most effective shRNA sequence (CTATGAGAAATTCTTCCTT) were selected based on the results of Western blotting and qRT-PCR in the preliminary experiment, which were named Lv-LPAR1shRNA. The viral particles containing the scrambled shRNA were named Lv-LPAR1-shRNA-NC.

The LPAR1 cDNA was ligated into a lentiviral vector, i.e., the GV492 plasmid, to amplify the LPAR1 gene. The LPAR1 gene was amplified using the forward primer gGGATCCCGCCACCATGGCTGCCATC and the reverse primer gACCGGTAACCACAGAGTGG. The PCR products were cleaved using BamH I and Ape I and ligated into the lentiviral vector (the GV492 plasmid).
The GV492-LPAR1 plasmid and other packaging plasmids were cotransfected into HEK293T cells using the same method described above, and the viral particles were named Lv-LPAR1. Additionally, a GV492 plasmid vector lacking the LPAR1 insert was also transfected into HEK293T cells to obtain a control virus (Lv-LPAR1-NC).

The cells were seeded in 24-well plates at a density of $5 \times 10^{4}$ cells per well. After incubating the cells for $24 \mathrm{~h}$, the lentiviral particles were diluted in enhanced infection solution at different multiplicities of infection, and polybrene $(5 \mu \mathrm{g} / \mathrm{ml})$ was added to the cells. The medium (RPMI-1640 supplemented with $10 \%$ FBS) was changed after $8-12 \mathrm{~h}$. At $72 \mathrm{~h}$ after infection, cells that had been infected with lentiviral vectors encoding enhanced green fluorescent protein (eGFP) were observed under a fluorescence microscope to detect the transduction efficiency and eGFP expression. Cells with $80 \%$ infection efficiency and good proliferation were selected and expanded in the presence of puromycin. The efficiency of the silencing and amplification of LPAR1 was confirmed using Western blotting and qRT-PCR.

\section{Xenograft experiments}

All procedures performed in animal studies were approved by the Animal Research Ethics Committee of Capital Medical University. Seventy-two female nude mice (female BALB/c, 4 weeks of age) were randomly divided into 12 groups (6 mice per group). After harvesting and resuspending tumor cells in phosphate-buffered saline, $3 \times 10^{6}$ cells were subcutaneously injected into the right flank of each nude mouse. The tumor width and length were measured every 5 days with digital calipers. The tumor volumes were calculated using the formula (width) ${ }^{2} \times$ length $/ 2$. The nude mice were sacrificed via $\mathrm{CO}_{2}$ inhalation after 30 to 60 days of observation, depending on the tumor growth rate. The tumors were isolated, fixed with $10 \%$ formalin, and embedded in paraffin for further pathological analyses.

\section{Statistical analysis}

Statistical analyses were performed using the SPSS 22.0 statistical package. The quantitative data are presented as mean \pm SD and were analyzed using ANOVA or twotailed Student's t-tests. The Wilcoxon signed rank test was used to compare the differences in LPAR1 expression between HGSC lesions obtained from two different sites in the same patients. The two-sample rank sum test was used to analyze the relationship between LPAR1 expression and the prognosis. A two-sided $\mathrm{P}$-value $<0.05$ was considered statistically significant. 
Table 1 Clinicopathological characteristics of the 74 patients with HGSC

\begin{tabular}{lll}
\hline & Mean or number & Range or percentage \\
\hline Age at diagnosis (years) & $53.41 \pm 9.29$ & $34-76$ \\
Menopausal status & & \\
Pre-menopausal & 23 & $31.1 \%$ \\
$\quad$ Post-menopausal & 51 & $68.9 \%$ \\
Family history of cancer & & \\
Yes & 6 & $8.1 \%$ \\
No & 68 & $91.9 \%$ \\
Preoperative CA125 levels & $2005.42 \pm 3137.71$ & $15-17,980$ \\
(IU/ml) & & \\
FIGO stage & 10 & $13.5 \%$ \\
Stage I & 6 & $8.1 \%$ \\
Stage II & 52 & $70.3 \%$ \\
Stage III & 6 & $8.1 \%$ \\
Stage IV & & \\
Lymphatic metastasis & 28 & $37.8 \%$ \\
Yes & 46 & $62.2 \%$ \\
No & & \\
Cytoreductive surgery & 53 & $71.6 \%$ \\
Residual tumor $\leq 1 \mathrm{~cm}$ & 53 & $29.7 \%$ \\
Residual tumor $>1 \mathrm{~cm}$ & 22 & \\
Current status & & $33.8 \%$ \\
NED & 25 & \\
AWD & 19 & \\
DOD & $30.5 \%$ \\
\hline
\end{tabular}

HGSC high-grade sercous carcinoma, FIGO International Federation of Gynecology and Obstetrics, NED no evidence of disease, AWD alive with disease, $D O D$ die of disease

\section{Results}

Heterogeneous expression of LPAR1 in human OSC tissues Seventy-four patients with OSC were included in the present study, and the clinicopathological characteristics of these patients are presented in Table 1 . The mean age at diagnosis was $53.41 \pm 9.29$ (range 34-76) years. All included patients were diagnosed with HGSC. Based on the FIGO 2014 staging system, 10, 6, 52, and 6 patients had stage I, stage II, stage III, and stage IV tumors, respectively. The TMAs included 166 OSC tissue specimens, including primary tumor lesions (74), abdominal disseminated lesions (52), lymphatic metastatic lesions (28) and recurrent lesions (12). The recurrent lesions included pelvic masses, lymph nodes, rectal tissues, and colon tissues, which were obtained through biopsy or open-abdominal surgery. The clinicopathological characteristics of these patients with recurrent OSC are presented in Table 2. The numbers of primary tumor lesions matching the abdominal disseminated lesions, lymphatic metastatic lesions and recurrent lesions from the same patients with OSC were 52,28 , and 12 , respectively. IHC staining was performed to detect LPAR1 expression in the matched specimens from the same patients. Compared with the primary tumor lesions, increased LPAR1 staining was observed in the recurrent and lymphatic metastatic lesions from the same patients (Fig. 1). The $\mathrm{H}$-scores for LPAR1 staining in the lymphatic metastatic lesions and recurrent lesions were noticeably higher than the primary tumor lesions $(159.08 \pm 27.23$ vs $145.69 \pm 29.45$, $\mathrm{P}=0.024 ; 165.25 \pm 21.49$ vs $145.69 \pm 29.45, \mathrm{P}=0.031$,

Table 2 The clinicopathological characteristics of these patients with recurrent HGSC

\begin{tabular}{llllll}
\hline Patient & $\begin{array}{l}\text { FIGO } \\
\text { stage }\end{array}$ & Recurrent site & $\begin{array}{l}\text { Recurrence } \\
\text { interval } \\
\text { (months) }\end{array}$ & $\begin{array}{l}\text { Recurrent lesions } \\
\text { obtained for IHC }\end{array}$ & $\begin{array}{l}\text { Method used to obtain } \\
\text { specimens }\end{array}$ \\
\hline 1 & IVB & Brain & 10 & Pelvic masses & Open-abdominal surgery \\
status
\end{tabular}

HGSC high-grade sercous carcinoma, FIGO International Federation of Gynecology and Obstetrics, IHC immunohistochemistry, DOD die of disease, AWD alive with disease 


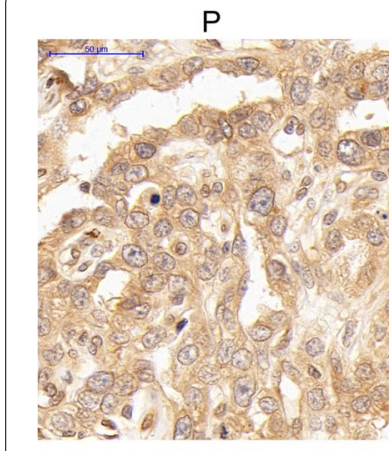

LN

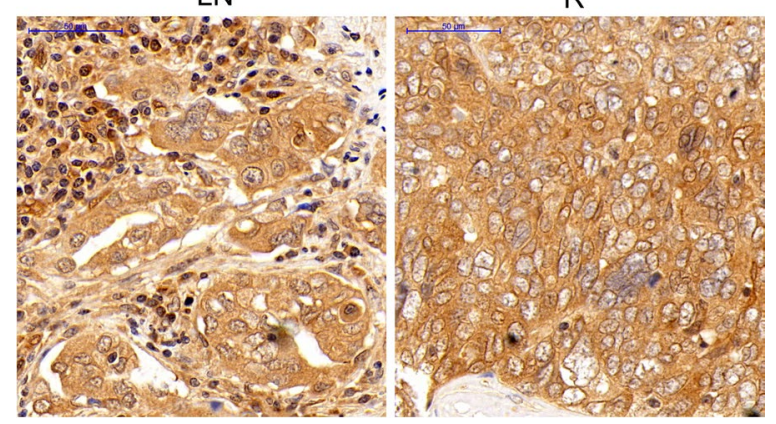

Fig. $1 \mathrm{IHC}$ staining for the LPAR1 protein in four types of matched lesions from a patient with OSC. In the image of $\mathrm{IHC}$ staining from the same patient, increased LPAR1 staining was observed in the recurrent lesions and lymphatic metastatic lesions compared with the primary tumor lesions. However, no differences in LPAR1 expression were observed between the primary tumor lesions and abdominal disseminated lesions. Original magnification: $\times 400 . P$ primary tumor samples, $A$ abdominal disseminated samples, $L N$ lymph node metastases samples, $R$ recurrent samples

Table 3 The H-scores for LPAR1 staining in four types of HGSC tissue specimens

\begin{tabular}{lll}
\hline & Number & LPAR1 H-score/ $\mathbf{P}^{\mathbf{a}}$ value \\
\hline Primary samples & 74 & $145.69 \pm 29.45$ \\
Abdominal disseminated samples & 52 & $147.79 \pm 30.64$ \\
Lymphatic metastatic samples & 28 & $159.08 \pm 27.23$ \\
$\begin{array}{l}\text { Recurrent samples } \\
\text { Primary samples vs disseminated }\end{array}$ & 12 & $165.25 \pm 21.49$ \\
$\quad$ samples & 52 & 0.152 \\
$\begin{array}{l}\text { Primary samples vs Lymphatic } \\
\quad \text { metastatic samples }\end{array}$ & 28 & 0.024 \\
$\begin{array}{l}\text { Primary samples vs Recurrent } \\
\quad \text { samples }\end{array}$ & 12 & 0.031 \\
\hline
\end{tabular}

Italic values indicate statistical significance $(P<0.05)$

HGSC high-grade sercous carcinoma, LPAR1 lysophosphatidic acid receptor 1

a Wilcoxon's signed rank test

respectively) (Table 3). However, no differences in the $\mathrm{H}$-scores for LPAR1 staining were not observed between the primary tumor lesions and abdominal disseminated lesions $(145.69 \pm 29.45$ vs $147.79 \pm 30.64, \mathrm{P}=0.152)$. In addition, the $\mathrm{H}$-score for LPAR1 staining in the primary tumor lesions was significantly higher in the patients with a PFS of less than 12 months or OS of less than 36 months $(\mathrm{P}=0.017 / 0.039)$, indicating that higher levels of the LPAR1 protein were associated with a worse prognosis (Table 4).

\section{Establishment of cell models of ITH in vitro}

Two pairs of single-cell subclones with distinct invasive/migratory capacities established in our previous study (named A-H/A-L/S-H/S-L) were seeded in two 96-well plates using the limiting dilution methodology. At 4 to $6 \mathrm{~h}$ after isolation and inoculation, single adherent cells were observed, and over time, the cell number continued to increase (Fig. 2a). The single-cell subclones were transferred from 96-well plates to 48-, 24-, and 6-well plates and, ultimately, $25-\mathrm{cm}$ square culture flasks within 6 to 10 weeks. Sixteen A-H, 13 A-L, $15 \mathrm{~S}-\mathrm{H}$, and $12 \mathrm{~S}-\mathrm{L}$ single-cell subclones were obtained. The single-cell subclones exhibiting the highest and lowest invasive/migratory capacities were selected based on the results from Transwell invasion/migration assays (Fig. 2b); these subclones were renamed A-H1 (A2780 high), A-L1 (A2780 low), S-H1 (SKOV3 high), and S-L1 (SKOV3 low). The Transwell invasion/migration assays revealed significantly greater invasive/migratory capacities of A-H1 cells than A-L1 cells (invasion: $550,839 \pm 62590$ vs $138,417 \pm 19,075, \mathrm{P}=0.003$; migration: $274,674 \pm 40,009$ vs $87,295 \pm 7186, \mathrm{P}=0.010$ ). Similarly, the S-H1 cells and S-L1 cells exhibited the most distinct differences in invasion/migration (invasion: $750,693 \pm 65,709$ vs $267,164 \pm 43,846, \mathrm{P}=0.004$; migration: $540,902 \pm 51,325$ vs $180,497 \pm 28,749$, $\mathrm{P}=0.004)$. The levels of the LPAR1 protein and mRNA were detected using Western blotting and qRT-PCR, respectively. The statistical analyses consistently revealed significantly increased levels of the LPAR1 mRNA and protein in the A-H1/S-H1 cells than in the A-L1/S-L1 cells (Fig. 2c) (qRT-PCR: $\mathrm{P}=0.004 /<0.001$; Western blotting: $\mathrm{P}=0.002 / 0.004)$.

\section{Establishment of stable LPAR1 knockdown and LPAR1-overexpressing cells}

Stable LPAR1 knockdown and LPAR1-overexpressing cell lines were established by transducing the cells with lentiviral particles. The A-H1 and S-H1 cells infected with Lv-LPAR1-shRNA were stable LPAR1 knockdown cells, which were named A-Lv-shLPAR1 and S-Lv-shLPAR1 cells, respectively. The A-H1 and $\mathrm{S}-\mathrm{H} 1$ cells infected with Lv-LPAR1-shRNA-NC were named A-Lv-shNC and S-Lv-shNC cells, respectively, and were considered 
Table 4 The relationship between LPAR1 expression and prognosis

\begin{tabular}{|c|c|c|c|c|c|c|}
\hline & $\begin{array}{l}\text { Primary samples } \\
n=74\end{array}$ & & $P$ value & $\begin{array}{l}\text { Primary samples } \\
n=74\end{array}$ & & $P$ value \\
\hline & $\begin{array}{l}\text { PFS }<12 \text { months } \\
n=16\end{array}$ & $\begin{array}{l}\text { PFS } \geq 12 \text { months } \\
n=58\end{array}$ & & $\begin{array}{l}\text { OS }<36 \text { months } \\
n=38\end{array}$ & $\begin{array}{l}O S \geq 36 \text { months } \\
n=36\end{array}$ & \\
\hline LPAR1 & $143.76 \pm 18.93$ & $136.65 \pm 23.34$ & $0.017^{\mathrm{a}}$ & $148.20 \pm 19.62$ & $137.43 \pm 26.67$ & $0.039^{\mathrm{a}}$ \\
\hline
\end{tabular}

Italic values indicate statistical significance $(P<0.05)$

LPAR1 lysophosphatidic acid receptor 1, PFS progression-free survival, OS overall survival

a Two samples rank sum test

the control groups for the knockdown groups. Similarly, the A-L1 and S-L1 cells infected with Lv-LPAR1 were LPAR1-overexpressing cells, which were named A-LvLPAR1 and S-Lv-LPAR1 cells, respectively. The A-L1 and S-L1 cells infected with Lv-LPAR1-NC were named A-Lv-NC and S-Lv-NC, respectively, and were considered the control groups for the overexpressing groups. At $72 \mathrm{~h}$ after infection, cells infected with plasmids containing eGFP were observed under a fluorescence microscope (Fig. 2d). qRT-PCR and Western blotting were conducted to detect LPAR1 levels. The statistical analyses consistently indicated significant and stable downregulation of the LPAR1 mRNA and protein in the LPAR1 knockdown cells and significant and stable upregulation of the LPAR1 mRNA and protein in the LPAR1-overexpressing cells (Fig. 2e, f) (all $\mathrm{P}<0.01)$. In addition, the control groups and the corresponding wild-type groups exhibited similar levels of the LPAR1 protein and mRNA (all P >0.05).

\section{Role of LPAR1 in biological functions in vitro}

According to the results of the Transwell invasion/ migration assays (Fig. 3a), invasion and migration of the LPAR1 knockdown groups were significantly decreased compared with the corresponding control groups (invasion: A2780: $732,395 \pm 39,154$ vs $467,818 \pm 36,623$, $\mathrm{P}=0.008$; SKOV3: $894,327 \pm 45,417$ vs $537,955 \pm 25,284$, $\mathrm{P}=0.002$ ) (migration: A2780: 598,600 $\pm 21,514 \quad$ vs $219,396 \pm 13,421, \quad \mathrm{P}<0.001 ; \quad S K O V 3: \quad 711,429 \pm 29,264$ vs $344,107 \pm 20,447, \mathrm{P}<0.001)$. Similarly, the invasion/ migration of the LPAR1-overexpressing groups were significantly increased compared with the corresponding control groups (invasion: A2780: $265,436 \pm 19,202$ vs $450,506 \pm 31,967, \mathrm{P}=0.008$, SKOV3: $362,365 \pm 22,484$ vs $567,782 \pm 47,373, \quad \mathrm{P}=0.017$ ) (migration: A2780: $152,081 \pm 33,230$ vs $468,313 \pm 22,950, \mathrm{P}=0.001$; SKOV3: $302,498 \pm 20,021$ vs $497,588 \pm 54,052, \mathrm{P}=0.028)$. A cell proliferation assay was performed using a CCK- 8 kit to detect the effect of LPAR1 on cell proliferation (Fig. 3b). Based on the cell growth curves, the LPAR1 deficiency inhibited the proliferation of the A-Lv-shLPAR1 and S-Lv-shLPAR1 cells $(48 \mathrm{~h}: \mathrm{P}=0.040 /<0.001 ; 72 \mathrm{~h}$ : $\mathrm{P}=0.005 / 0.004)$. In contrast, the LPAR1 overexpression accelerated the proliferation of the A-Lv-LPAR1 and S-Lv-LPAR1 cells ( $48 \mathrm{~h}: \mathrm{P}=0.001 / 0.002 ; 72 \mathrm{~h}$ : $\mathrm{P}=0.039 / 0.004)$. In addition, differences in invasion, migration, and proliferation were not observed between the control groups and the corresponding wild-type groups (all $\mathrm{P}>0.05$ ).

\section{Role of LPAR1 in tumor formation in vivo}

Xenograft experiments were performed to test and verify the role of LPAR1 in tumor formation. Twelve groups of nude mice were injected with A-H1-wild-type, A-LvshNC, A-Lv-shLPAR1, A-L1-wild-type, A-Lv-NC, A-LvLPAR1, S-H1-wild-type, S-Lv-shLPAR1, S-Lv-shNC, S-L1-wild-type, S-Lv-NC, and S-Lv-LPAR1 cells (Fig. 4a). At the end of the observation period (45 days for the A2780 cells and 60 days for the SKOV3 cells), the tumor formation rates in the mice injected with A-L1-wildtype, A-Lv-shNC, S-L1-wild-type and S-Lv-shNC cells were $33.3 \%(2 / 6), 50 \%(3 / 6), 83.3 \%(5 / 6)$, and $66.7 \%(4 / 6)$, respectively, and the rate in the other groups was $100 \%$. Compared with the corresponding control groups, the A-Lv-shLPAR1 and S-Lv-shLPAR1 groups had tumors with significantly smaller volumes $(\mathrm{P}<0.001 /=0.001)$ and weights (both $\mathrm{P}<0.001$ ). In contrast, the tumors in the A-L1-LPAR1 and S-L1-LPAR1 groups were much larger than in the corresponding control groups in terms of both volume (both $\mathrm{P}<0.001$ ) and weight (both $\mathrm{P}<0.001$ ). In addition, no differences were observed between the control groups and the corresponding wild-type groups (all $\mathrm{P}>0.05$ ). The tumor tissues were fixed with formalin, embedded in paraffin, and stained with H\&E and IHC to detect LPAR1 expression (Fig. 4b). LPAR1 expression was detected using IHC, which verified the efficiency of the transduction with the lentiviral particles.

\section{Correlation between LPAR1 expression and the PI3K/AKT pathway}

The levels of PI3K p85 alpha phosphorylated at Y607 $\left(\mathrm{p}-\mathrm{PI} 3 \mathrm{~K} \quad \mathrm{Y}^{607}\right)$ and AKT1/2/3 phosphorylated at S472 + S473 + S474 (p-AKT S $\left.{ }^{472 / 473 / 474}\right)$ in ITH cell models with stable LPAR1 knockdown and LPAR1 overexpression were detected using Western blotting to explore 


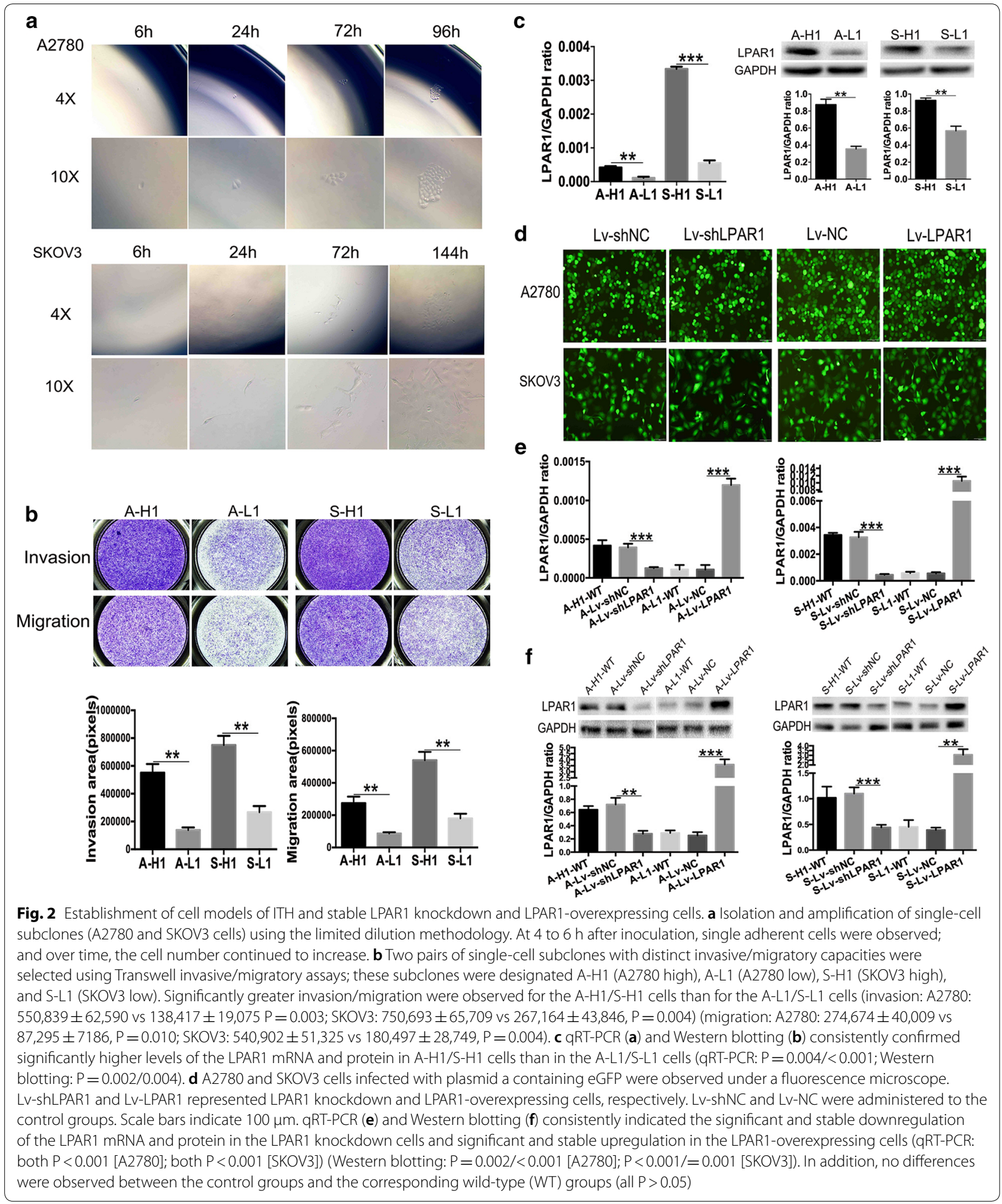

the correlation between LPAR1 expression and the PI3K/ AKT pathway (Fig. 3c). Western blot analyses revealed that significantly decreased levels of p-PI3K and p-AKT in the LPAR1 knockdown cells ( $\mathrm{p}-\mathrm{PI} 3 \mathrm{~K}$ : $\mathrm{P}=0.003 / 0.009$;
p-AKT: $P=0.040 / 0.010)$ and significantly increased levels in the LPAR1-overexpressing cells (p-PI3K: $\mathrm{P}=0.003 / 0.016 ; \quad \mathrm{p}-\mathrm{AKT}: \mathrm{P}=0.017 / 0.006)$ compared with the corresponding control groups. In addition, no 


\begin{abstract}
(See figure on next page.)
Fig. 3 Role of LPAR1 in modulating biological functions and the correlation between LPAR1 expression and the PI3K/AKT pathway. a Transwell invasion/migration assays revealed significant decreases in the invasion and migration of the LPAR1 knockdown groups compared with the corresponding control groups (invasion: A2780: $732,395 \pm 39,154$ vs 467,818 $\pm 36,623, P=0.008$; SKOV3: 894,327 $\pm 45,417$ vs $537,955 \pm 25,284$, $P=0.002$ ) (migration: A2780: 598,600 $\pm 21,514$ vs 219,396 $\pm 13,421, P<0.001 ;$ SKOV3: 711,429 $\pm 29,264$ vs 344,107 $\pm 20,447, P<0.001$ ). Similarly, the invasion/migration of the LPAR1-overexpressing cells were significantly increased compared with the corresponding control groups (invasion: A2780: $265,436 \pm 19,202$ vs 450,506 $\pm 31,967, P=0.008$, SKOV3: $362,365 \pm 22,484$ vs 567,782 $\pm 47,373, P=0.017$ ) (migration: A2780: $152,081 \pm 33,230$ vs $468,313 \pm 22,950, \mathrm{P}=0.001$; SKOV3: $302,498 \pm 20,021$ vs $497,588 \pm 54,052, \mathrm{P}=0.028)$. b A cell proliferation assay was performed using a CCK-8 kit, and the cell growth curves showed that the LPAR1 deficiency inhibited the proliferation of the A-LV-shLPAR1 and S-LV-shLPAR1 cells (48 h: $P=0.040 /<0.001 ; 72 \mathrm{~h}: \mathrm{P}=0.005 / 0.004)$. In contrast, LPAR1 overexpression accelerated the proliferation of the A-LV-LPAR1 and S-LV-LPAR1 cells (48 $\mathrm{h}: \mathrm{P}=0.001 / 0.002 ; 72 \mathrm{h:} \mathrm{P}=0.039 / 0.004$ ). In addition, differences in the invasion, migration, and proliferation were not observed between the control groups and the corresponding wild-type (WT) groups (all P >0.05). c Western blot analyses revealed significantly decreased levels of PI3K p85 alpha phosphorylated at Y607 (p-PI3K Y ${ }^{607}$ ) and AKT1/2/3 phosphorylated at $\$ 472+S 473+$ S474 (p-AKT S ${ }^{472 / 473 / 474}$ ) in the LPAR1 knockdown cells ( $p$-PI3K: $P=0.003 / 0.009 ; p-A K T: P=0.040 / 0.010)$ and significantly increased levels in the LPAR1-overexpressing cells $(p-P I 3 K: P=0.003 / 0.016$; $\mathrm{P}$-AKT: $P=0.017 / 0.006$ ). In addition, no differences were observed between the control groups and the corresponding wild-type groups (all $P>0.05)$
\end{abstract}

differences were observed between the control groups and the corresponding wild-type groups (all $\mathrm{P}>0.05$ ).

\section{Discussion}

A heterogeneous mixture of functionally distinct cancer cells exhibiting varying levels of receptor activity and differentiation and distinct metabolic and epigenetic states exists within a tumor [6]. ITH leads to wide range of responses of tumors to therapeutic agents, resulting in many difficulties in clinical treatment. ITH in ovarian cancer, which has been reported in the literature $[9,23]$ and our previous study [11], is related to tumor metastasis, chemotherapy resistance and recurrence. In the present study, TMAs including four types of matched primary and recurrent tumor lesions obtained from the same patients with OSC were constructed to explore ITH in OSC. In tissues from the same patients, the levels of the LPAR1 protein were noticeably higher in the lymphatic metastatic and recurrent OSC tissues than in the primary tumor lesions. Based on these results, ITH exists in OSC and LPAR1 plays an essential role in the development of ITH. In addition, the LPAR1 protein is expressed at high levels in patients with less than 12 months of PFS or 36 months of OS, as detected by IHC staining. Similarly, Yu et al. [24] reported significantly increased LPAR1 expression in patients with advanced clinical stages of epithelial ovarian cancer, an abdominal metastasis of more than $2 \mathrm{~cm}$, retroperitoneal lymph node metastasis or hepatic metastasis. Based on the previous study and our data, we propose that high expression of LPAR1 represents a potential predictor of a poor prognosis for patients with OSC.

LPAR1 plays as an important role in the development of malignant tumors, including breast cancer, ovarian cancer, and pancreatic cancer, by binding to LPA and activating downstream targets $[18,25,26]$. In cancer cells, LPAR1 contributes to DNA synthesis and cell division following lipid phosphate phosphatase-1 and LPA stimulation [27]. According to Park et al. [28], LPAR1 mediates the LPA-induced migration of ovarian cancer cells. Silencing of LPAR1 alone in HEY and SKOV3 cells significantly reduces LPA-induced invasion [24]. The ovarian cancer stem cell properties induced by LPA stimulation are abrogated by LPAR1-specific inhibitors or LPAR1 silencing [29]. In addition, LPAR1 plays an essential role in the progression of bone metastasis, and antagonists blocking the LPAR1-dependent effects of LPA represent potential therapeutic targets in these patients [30]. Although accumulating evidence have revealed a role for LPAR1 in malignant tumors, few studies have focused on the role of LPAR1 in the development of ITH. In our previous study, we observed significantly increased levels of the LPAR1 protein and mRNA in ITH cell models with high invasive/migratory capacities [11]. Single-cell subclones with distinct invasive/migratory capacities were reisolated, and cell models of ITH were reidentified in this study to explore and verify the role of LPAR1 in the development of ITH. In vitro biological functions, including invasive, migratory, and proliferative capacities, and in vivo tumor formation were significantly decreased in the LPAR1-silenced ITH cell models. In contrast, cellular functions were significantly enhanced in the LPAR1-overexpressing ITH cell models both in vitro and in vivo. Thus, LPAR1 plays a vital role in regulating the viability of independent heterogeneous subsets of OSC cell lines, and the development of ITH in OSC is strongly correlated with LPAR1 expression.

As shown in our previous study, the activation of the $\mathrm{PI} 3 \mathrm{~K} / \mathrm{AKT} / \mathrm{mTOR}$ pathway is associated with the development of ITH in human OSC [11]. As a member of the GPCR superfamily, LPAR1 activates the PI3K/AKT pathway by binding to $G_{\alpha i / o}$ proteins [13]. One of the most notable functions of LPAR1-induced activation of $\mathrm{G}_{\mathrm{\alpha i} / \mathrm{o}}$ proteins in rodent Schwann cells is survival signaling via the PI3K/AKT pathway [16]. LPA induces hyaluronic acid synthesis in human skin fibroblasts mainly by activating 


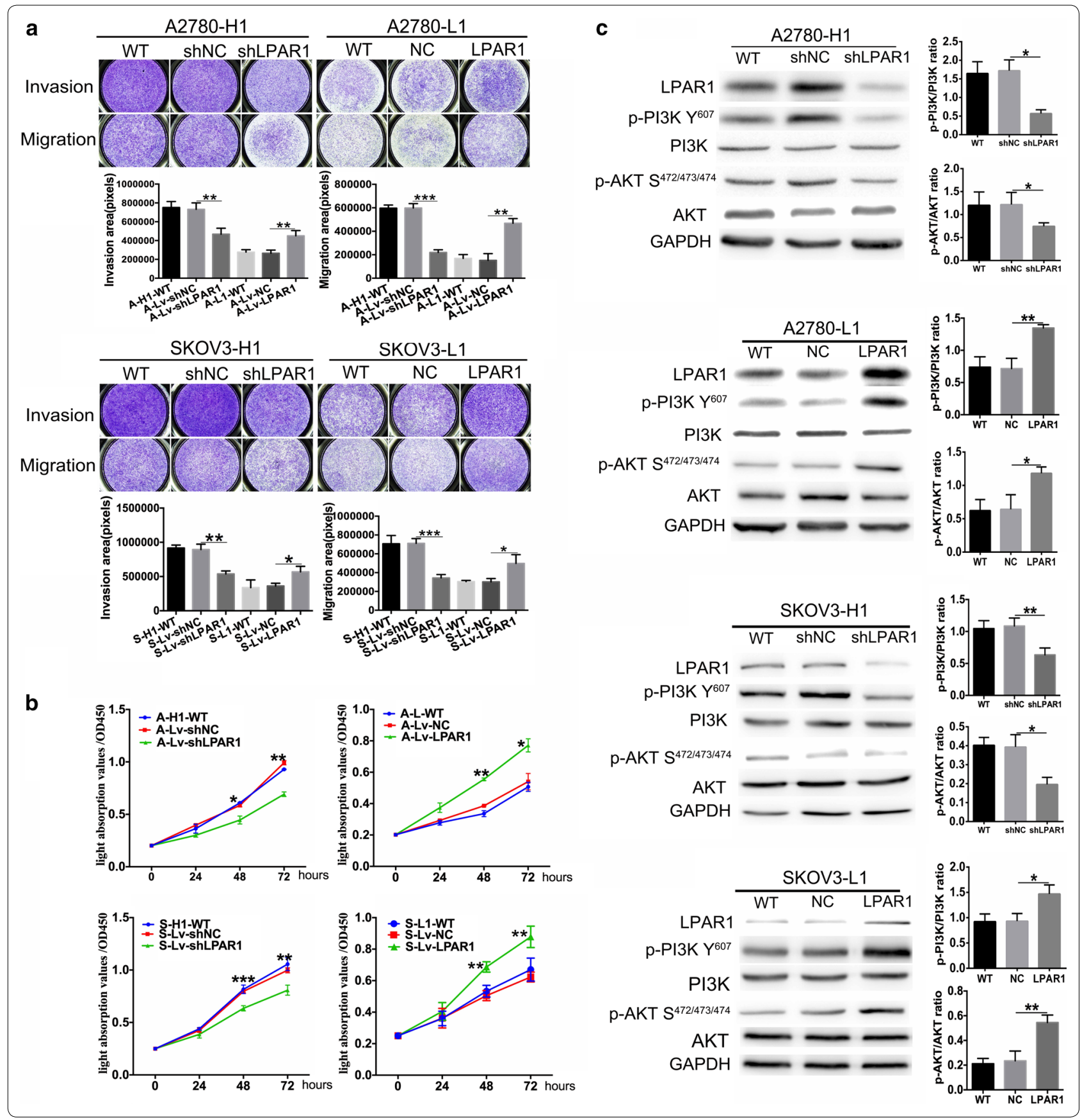

(See figure on next page.)

Fig. 4 Role of LPAR1 in tumor formation in vivo. Xenograft experiments were performed to test and verify the role of LPAR1 in tumor formation. a Compared with the corresponding control groups, the tumors in the A-Lv-shLPAR1 and S-Lv-shLPAR1 groups were significantly smaller in terms of both volume $(P<0.001 /=0.001)$ and weight (both $P<0.001)$. In contrast, the tumors in the A-L1-LPAR1 and S-L1-LPAR1 groups were much larger than in the corresponding control groups in terms of both volume (both $\mathrm{P}<0.001$ ) and weight (both $\mathrm{P}<0.001$ ). In addition, no difference was observed between the control groups and the corresponding wild-type (WT) groups (all P>0.05). $\mathbf{b}$ The tumor tissues were fixed formalin, embedded in paraffin, and stained with H\&E. IHC were performed to detect LPAR1 expression. LPAR1 expression was detected using IHC, which verified the efficiency of the transduction with the lentiviral particles 
a
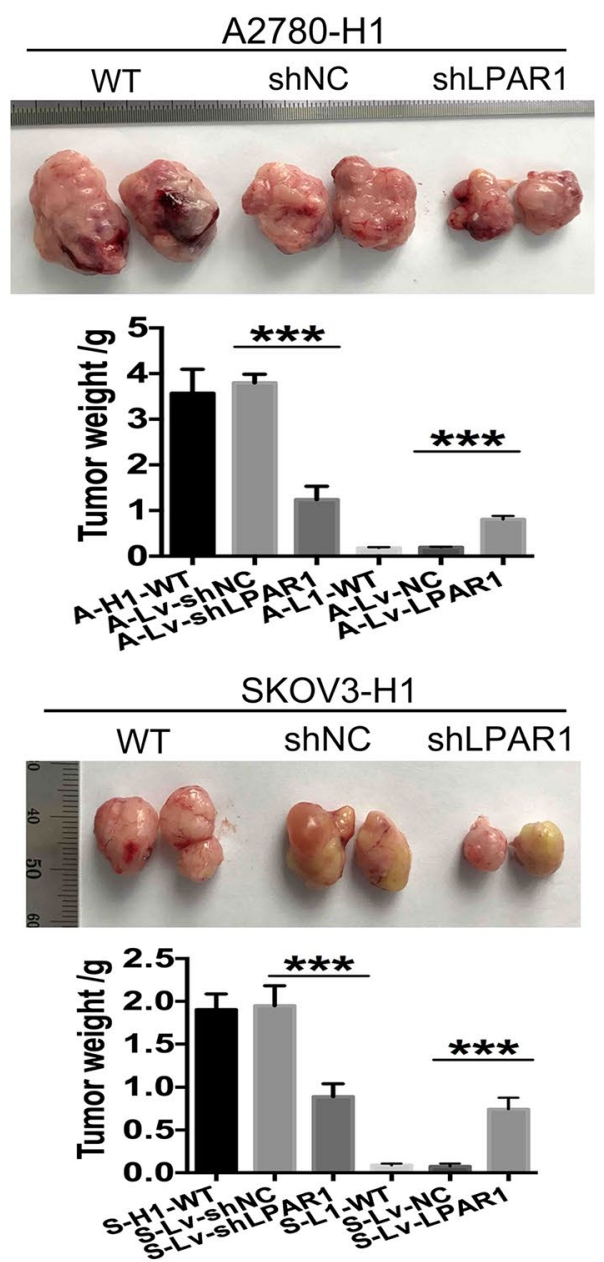

b

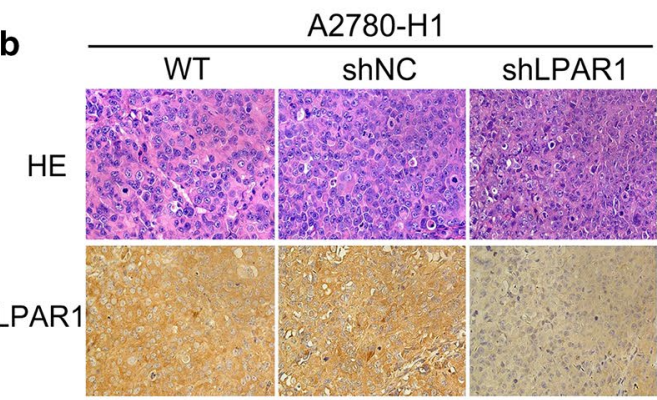

SKOV3-H1

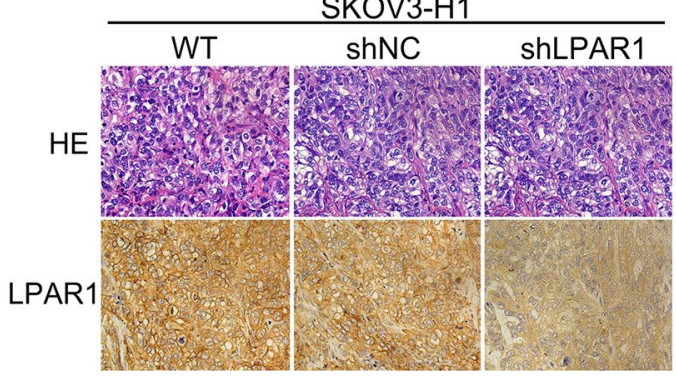

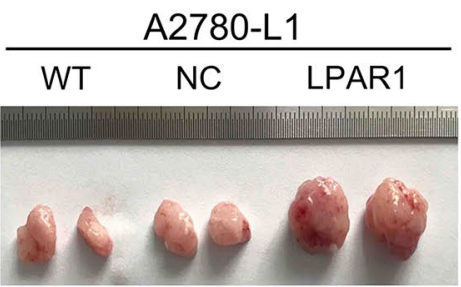

릉 $\left.{ }^{4}\right] T^{\star \star * *}$

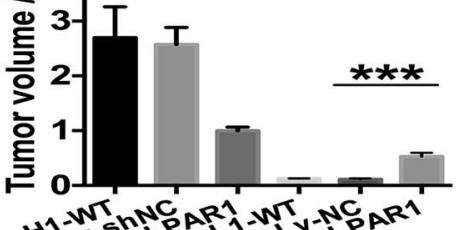

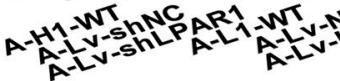
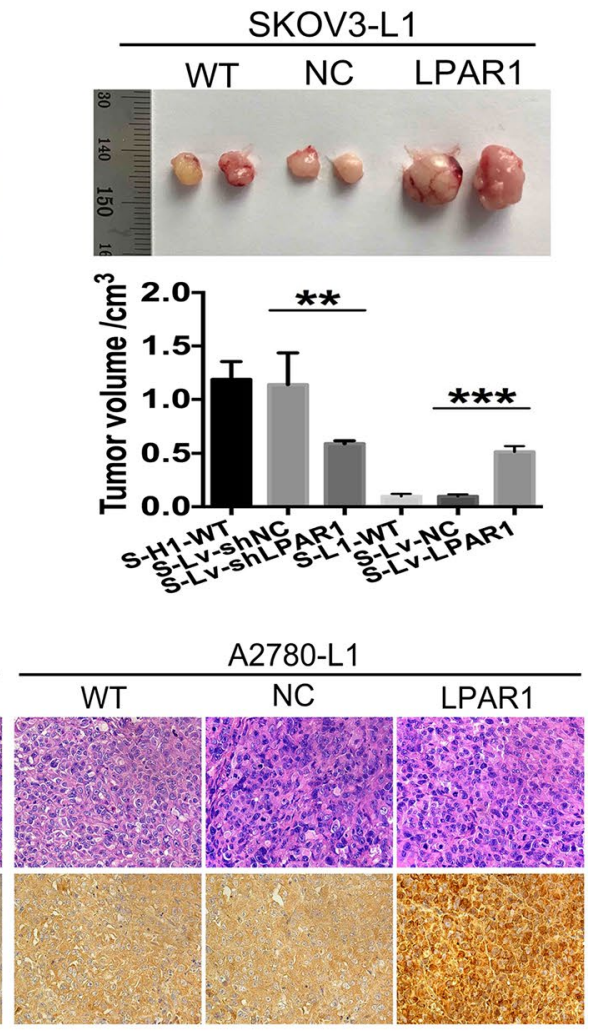

SKOV3-L1

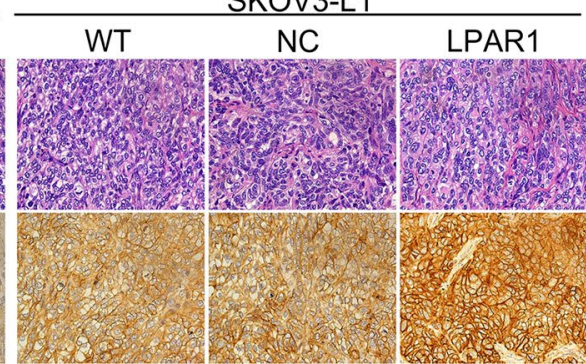


LPAR1- $\mathrm{G}_{\mathrm{\alpha i} / \mathrm{o}}$, followed by the activation of the PI3K signaling pathway [17]. Sahay et al. [18] reported that LPAR1/ PI3K signaling mediates the LPA-dependent metastasis of breast cancer cells. However, to the best of our knowledge, data related to the regulatory effect of LPAR1 on the PI3K/AKT pathway in OSC are lacking. We detected the levels of p-PI3K and p-AKT in LPAR1-silenced and LPAR1-overexpressing ITH cell models to investigate the correlation between LPAR1 expression and the $\mathrm{PI} 3 \mathrm{~K} / \mathrm{AKT}$ pathway and determine the potential mechanisms involved in the development of ITH. The levels of p-PI3K and p-AKT were significantly decreased in the LPAR1 knockdown cells and significantly increased in the LPAR1-overexpressing cells. Based on these results, LPAR1 might be involved in the development of ITH in human OSC by activating the PI3K/AKT signaling pathway. Activation of the PI3K/AKT pathway is involved in carcinogenesis and ovarian cancer development [31, 32]. Certain experts have suggested that PI3K only plays a passive role in the development of human malignancies $[33,34]$. The inhibition of PI3K potentially leads to feedback upregulation of the expression and phosphorylation of multiple receptor tyrosine kinases [35]. Researchers have proposed that PI3K inhibitors should be used in combination with other antagonists in cancer therapy $[35,36]$. As shown in the present study, the levels of $\mathrm{p}-\mathrm{PI} 3 \mathrm{~K}$ and $\mathrm{p}-\mathrm{AKT}$ vary according to the expression of the LPAR1 protein. LPAR1, which is located upstream of the PI3K/AKT pathway, may present an effective target for the treatment of ovarian cancer.

Our study provides the first evidence of roles for LPAR1 and the PI3K/AKT pathway in ITH, and a potential treatment target for OSC has been described. However, some important limitations also exist in the present study. First, IHC staining for LPAR1 in human OSC tissues must be interpreted with caution due to the drawbacks of TMAs. TMAs including the limited tissues collected in the present study might not be representative of the whole tumor. In addition, due to the limitations of basic research, the effects of LPAR 1 and its correlation with the PI3K/AKT pathway should be verified in the future.

\section{Conclusions}

ITH exists in OSC, as evidenced by heterogeneous LPAR1 expression in primary, lymphatic metastatic and recurrent OSC tissue from the same patients. Higher levels of the LPAR1 protein were associated with a poor prognosis. LPAR1 plays essential roles in the invasion, migration, and proliferation of heterogeneous subsets of OSC cell lines and the development of ITH in OSC, possibly by activating the PI3K/AKT signaling pathway. Strategies that decrease LPAR1 expression represent a potential therapeutic target for OSC.

\section{Additional files}

Additional file 1: Table S1. The principle materials and instruments used in the present study.

Additional file 2: Table S2. Details of primary antibodies used for Western blotting.

Additional file 3: Table S3. The sequences of three specific shRNAs targeting the LPAR1 gene and the scrambled shRNA.

\section{Abbreviations}

ITH: intratumoral heterogeneity; OSC: ovarian serous cystadenocarcinoma; LPAR1: lysophosphatidic acid receptor 1; LPA: lysophosphatidic acid; GPCR: G protein-coupled receptor; FIGO: Federation of Gynecology and Obstetrics; PFS: progression-free survival; OS: overall survival; TMA: tissue microarray; $\mathrm{H}$-score: histochemistry score; FBS: fetal bovine serum; CCK-8: counting Kit-8; eGFP: enhanced green fluorescent protein; qRT-PCR: quantitative real-time polymerase chain reaction; $\mathrm{p}$-PI3K: phosphorylated PI3K; $\mathrm{P}$-AKT: phosphorylated AKT.

\section{Acknowledgements}

Not applicable.

\section{Authors' contributions}

RC, ZYZ and HMB: conception and design of the study, assembly, analysis and interpretation of the data, and manuscript writing. GMC: provision of study materials, analysis and interpretation of the data. All authors read and approved the final manuscript.

\section{Funding}

This work was supported by the Beijing Natural Science Foundation (No.7172077) and Beijing Municipal Administration of Hospitals Clinical Medicine Development of Special Funding-YangFan Project (No. ZYLX201713).

\section{Availability of data and materials}

The datasets used and/or analysed during the current study are available from the corresponding author on reasonable request.

\section{Ethics approval and consent to participate}

Ethical approval for the study was granted by the Medical Ethics Committee of Beijing Chao-Yang Hospital in accordance with the Declaration of Helsinki, and the written informed consent was obtained from each participant involved. Xenograft experiments were approved by Animal Research Ethics Committee of Capital Medical University, in accordance with the guidelines of Directive 2010/63/EU in Europe.

\section{Consent for publication}

Not applicable.

\section{Competing interests}

The authors declare that they have no competing interests.

Received: 31 March 2019 Accepted: 22 July 2019

Published online: 29 July 2019

\section{References}

1. Cheaib B, Auguste A, Leary A. The PI3K/Akt/mTOR pathway in ovarian cancer: therapeutic opportunities and challenges. Chin J Cancer. 2015;34(1):4-16.

2. Armstrong D. Update on treatment options for newly diagnosed ovarian cancer. Clin Adv Hematol Oncol H\&O. 2010;8(10):675-8.

3. Kang KW, Lee MJ, Song JA, Jeong JY, Kim YK, Lee C, et al. Overexpression of goosecoid homeobox is associated with chemoresistance and poor prognosis in ovarian carcinoma. Oncol Rep. 2014;32(1):189-98.

4. Khalique L, Ayhan A, Weale ME, Jacobs IJ, Ramus SJ, Gayther SA. Genetic intra-tumour heterogeneity in epithelial ovarian cancer and its implications for molecular diagnosis of tumours. J Pathol. 2007;211(3):286-95. 
5. Allison KH, Sledge GW. Heterogeneity and cancer. Oncology (Williston Park, NY). 2014;28(9):772-8.

6. Pribluda A, de la Cruz CC, Jackson EL. Intratumoral Heterogeneity: from Diversity Comes Resistance. Clin Cancer Res. 2015;21(13):2916-23.

7. Ruiz-Cerda JL, Hernandez M, Sempere A, O'Connor JE, Kimler BF, JimenezCruz F. Intratumoral heterogeneity of DNA content in renal cell carcinoma and its prognostic significance. Cancer. 1999;86(4):664-71.

8. Gerlinger M, Swanton C. How Darwinian models inform therapeutic failure initiated by clonal heterogeneity in cancer medicine. Br J Cancer. 2010;103(8):1139-43.

9. Abelson S, Shamai Y, Berger L, Shouval R, Skorecki K, Tzukerman M. Intratumoral heterogeneity in the self-renewal and tumorigenic differentiation of ovarian cancer. Stem Cells. 2012;30(3):415-24.

10. Gerlinger M, Rowan AJ, Horswell S, Math M, Larkin J, Endesfelder D, et al. Intratumor heterogeneity and branched evolution revealed by multiregion sequencing. N Engl J Med. 2012;366(10):883-92.

11. Bai H, Li H, Li W, Gui T, Yang J, Cao D, et al. The PI3K/AKT/mTOR pathway is a potential predictor of distinct invasive and migratory capacities in human ovarian cancer cell lines. Oncotarget. 2015;6(28):25520-32.

12. Chen J, Zhang J, Zhao Y, Li J, Fu M. Integrin beta3 down-regulates invasive features of ovarian cancer cells in SKOV3 cell subclones. J Cancer Res Clin Oncol. 2009;135(7):909-17.

13. Choi JW, Herr DR, Noguchi K, Yung YC, Lee CW, Mutoh T, et al. LPA receptors: subtypes and biological actions. Annu Rev Pharmacol Toxicol. 2010;50:157-86.

14. Fukushima N, Ishii I, Contos JJ, Weiner JA, Chun J. Lysophospholipid receptors. Annu Rev Pharmacol Toxicol. 2001:41:507-34.

15. Ishii I, Fukushima N, Ye X, Chun J. Lysophospholipid receptors: signaling and biology. Annu Rev Biochem. 2004:73:321-54.

16. Ye X, Ishii I, Kingsbury MA, Chun J. Lysophosphatidic acid as a novel cell survival/apoptotic factor. Biochem Biophys Acta. 2002;1585(2-3):108-13.

17. Maeda-Sano K, Gotoh M, Morohoshi T, Someya T, Murofushi H, MurakamiMurofushi K. Cyclic phosphatidic acid and lysophosphatidic acid induce hyaluronic acid synthesis via CREB transcription factor regulation in human skin fibroblasts. Biochem Biophys Acta. 2014;1841(9):1256-63.

18. Sahay D, Leblanc R, Grunewald TG, Ambatipudi S, Ribeiro J, Clezardin P, et al. The LPA1/ZEB1/miR-21-activation pathway regulates metastasis in basal breast cancer. Oncotarget. 2015;6(24):20604-20.

19. Prat J. FIGO's staging classification for cancer of the ovary, fallopian tube, and peritoneum: abridged republication. J Gynecol Oncol. 2015;26(2):87-9.

20. Liu M, Cao G, Zhang Y, Qu J, Li W, Wan X, et al. Changes in the morphology and protein expression of germ cells and Sertoli cells in plateau pikas testes during non-breeding season. Scientific Rep. 2016;6:22697.

21. Azim HA Jr, Peccatori FA, Brohee S, Branstetter D, Loi S, Viale G, et al. RANK-ligand (RANKL) expression in young breast cancer patients and during pregnancy. Breast Cancer Res BCR. 2015;17:24.

22. Zhang L, Xu J, Zhang $X$, Zhang $Y$, Wang L, Huang $X$, et al. The Role of Tumoral FOXP3 on Cell Proliferation, Migration, and Invasion in Gastric Cancer. Cell Physiol Biochem Int J Exp Cell Physiol Biochemis Pharmacol. 2017:42(5):1739-54.
23. Gui T, Bai H, Zeng J, Zhong Z, Cao D, Cui Q, et al. Tumor heterogeneity in the recurrence of epithelial ovarian cancer demonstrated by polycomb group proteins. Onco Targets Ther. 2014;7:1705-16.

24. Yu X, Zhang Y, Chen H. LPA receptor 1 mediates LPA-induced ovarian cancer metastasis: an in vitro and in vivo study. BMC Cancer. 2016;16(1):846.

25. Yamada T, Sato K, Komachi M, Malchinkhuu E, Tobo M, Kimura T, et al. Lysophosphatidic acid (LPA) in malignant ascites stimulates motility of human pancreatic cancer cells through LPA1. J Biol Chem. 2004:279(8):6595-605.

26. Liu S, Umezu-Goto M, Murph M, Lu Y, Liu W, Zhang F, et al. Expression of autotaxin and lysophosphatidic acid receptors increases mammary tumorigenesis, invasion, and metastases. Cancer Cell. 2009;15(6):539-50.

27. Xu J, Love LM, Singh I, Zhang QX, Dewald J, Wang DA, et al. Lipid phosphate phosphatase-1 and $\mathrm{Ca} 2+$ control lysophosphatidate signaling through EDG-2 receptors. J Biol Chem. 2000;275(36):27520-30.

28. Park J, Jang JH, Oh S, Kim M, Shin C, Jeong M, et al. LPA-induced migration of ovarian cancer cells requires activation of ERM proteins via LPA1 and LPA2. Cell Signal. 2018;44:138-47.

29. Seo EJ, Kwon YW, Jang IH, Kim DK, Lee SI, Choi EJ, et al. Autotaxin regulates maintenance of ovarian cancer stem cells through lysophosphatidic acid-mediated autocrine mechanism. Stem cells (Dayton, Ohio). 2016;34(3):551-64.

30. Boucharaba A, Serre CM, Guglielmi J, Bordet JC, Clezardin P, Peyruchaud O. The type 1 lysophosphatidic acid receptor is a target for therapy in bone metastases. Proc Natl Acad Sci USA. 2006;103(25):9643-8.

31. Knuefermann C, Lu Y, Liu B, Jin W, Liang K, Wu L, et al. HER2/PI-3K/Akt activation leads to a multidrug resistance in human breast adenocarcinoma cells. Oncogene. 2003;22(21):3205-12.

32. Gao N, Flynn DC, Zhang Z, Zhong XS, Walker V, Liu KJ, et al. G1 cell cycle progression and the expression of G1 cyclins are regulated by PIBK/AKT/ mTOR/p70S6K1 signaling in human ovarian cancer cells. Am J Physiol Cell Physiol. 2004;287(2):C281-91.

33. Bader AG, Kang S, Vogt PK. Cancer-specific mutations in PIK3CA are oncogenic in vivo. Proc Natl Acad Sci USA. 2006;103(5):1475-9.

34. Gymnopoulos M, Elsliger MA, Vogt PK. Rare cancer-specific mutations in PIK3CA show gain of function. Proc Natl Acad Sci USA. 2007;104(13):5569-74.

35. Chakrabarty A, Sanchez V, Kuba MG, Rinehart C, Arteaga CL. Feedback upregulation of HER3 (ErbB3) expression and activity attenuates antitumor effect of PI3K inhibitors. Proc Natl Acad Sci USA. 2012;109(8):2718-23.

36. Glaysher S, Bolton LM, Johnson P, Atkey N, Dyson M, Torrance C, et al. Targeting EGFR and PI3K pathways in ovarian cancer. Br J Cancer. 2013;109(7):1786-94.

\section{Publisher's Note}

Springer Nature remains neutral with regard to jurisdictional claims in published maps and institutional affiliations.

Ready to submit your research? Choose BMC and benefit from

- fast, convenient online submission

- thorough peer review by experienced researchers in your field

- rapid publication on acceptance

- support for research data, including large and complex data types

- gold Open Access which fosters wider collaboration and increased citations

- maximum visibility for your research: over 100M website views per year

At $B M C$, research is always in progress.

Learn more biomedcentral.com/submissions 